GANIT J. Bangladesh Math. Soc. (ISSN 1606-3694) 36 (2016) 91-112

\title{
SLIP VELOCITY DISTRIBUTION ON MHD OSCILLATORY HEAT AND MASS TRANSFER FLOW OF A VISCOUS FLUID IN A PARALLEL PLATE CHANNEL
}

\author{
Venkateswarlu Malapati $^{1{ }^{*}}$ and Venkata Lakshmi Dasari ${ }^{2}$ \\ ${ }^{1}$ Department of Mathematics, V. R. Siddhartha Engg College, Vijayawada, \\ Krishna (Dist), A.P, India, Pin-520 007. \\ ${ }^{2}$ Department of Mathematics, Bapatla Women's Engg College, Bapatla, \\ Guntur (Dist), A.P, India, Pin-522 102. \\ *Corresponding Author: mvsr2010@gmail.com
}

Received 24.07.2016 Accepted 01.10.2016

\begin{abstract}
The present investigation deals with the effect of slip on the hydromagnetic pulsatile flow through a parallel plate channel filled with saturated porous medium. Based on the pulsatile flow nature, the transformed conservation equations are solved analytically subject to physically appropriate boundary conditions by using two term perturbation technique. Exact solutions are obtained for the velocity, temperature and concentration fields. In particular skin friction coefficient, Nusselt number and Sherwood number are found to evolve into their steady state case in the large time limit. The results obtained here may be further used to verify the validity of obtained numerical solutions for more complicated transient free convection fluid flow problems. Parametric study of the solutions are conducted and discussed.
\end{abstract}

Keywords: MHD fluid, Navier slip, porous medium, thermal radiation, heat transfer.

\section{Introduction}

At the macroscopic level it is well accepted that the boundary condition for a viscous fluid at a solid wall is one of "no-slip", i.e., the fluid velocity matches the velocity of the solid boundary. While the no-slip boundary condition has been proven experimentally to be accurate for a number of macroscopic flows, it remains on assumption that is not based on physical principles. In fact, nearly two hundred years ago Navier [19] proposed a general boundary condition that incorporates the possibility of fluid slip at a solid boundary. Navier's proposed condition assumes that the velocity $v_{x}$ at a solid surface is proportional to the shear stress at the surface

$$
v_{x}=\gamma \frac{d v_{x}}{d y}
$$

where $\gamma$ is the slip strength or slip coefficient. If $\gamma=0$ then the general assumed no-slip boundary condition is obtained. If $\gamma$ is finite, fluid slip occurs at the wall but its effect depends upon the length scale of the flow. The above relation states that the velocity of the fluid at the plates is 
linearly proportional to the shear stress at the plate. Yu and Ameel [30] investigated on slip-flow heat transfer in rectangular micro-channels.

The fluid slippage phenomenon at the solid boundaries appear in many applications such as in microchannels or nanochannels and in applications where a thin film of light oil is attached to the moving plates or when the surface is coated with special coatings such as thick monolayer of hydrophobic octadecyltrichlorosilane. Derek et al. [4] presented apparent fluid slip at hydrophobic micro-channel walls. Also, wall slip can occur in the working fluid contains concentrated suspensions. Soltani and Yilmazer [23] discussed on slip velocity and slip layer thickness in flow of concentrated suspensions. However, the literature lacks studies that take into account the possibility of fluid slippage at the wall under vibrating conditions. Recently, several researchers have suggested that the no-slip boundary condition may not be suitable for hydrophilic flows over hydrophobic boundaries at both the micro and nano scale. Watanebe et al. [29] investigated on slip of Newtonian fluids at solid boundary. Ruckenstein and Rajora [21] investigated on the no-slip boundary conditions of hydrodynamics. Coutte flow with slip and jump boundary conditions under steady state conditions and only for gases have been investigated by Marques et al. [14]. The closed form solution for steady periodic and transient velocity field under slip condition have been studied by Khaled and Vafai [11]. The effect of slip condition on MHD steady flow in a channel with permeable boundaries has been discussed by Makinde and Osalusi [13]. Manjula et al. [15] presented the influence of thermal radiation and chemical reaction on MHD flow, heat and mass transfer over a stretching surface.

Studies related to the oscillatory fluid flow are increasingly important in recent times due to its numerous applications in many real life problems. Some of these include, Makinde [12] studied the combined effects of radiative heat transfer and magnetohydrodynamics on oscillatory flow in a channel filled with porous medium. Venkateswarlu and Padma [25] considered the unsteady MHD free convective heat and mass transfer in a boundary layer flow past a vertical permeable plate with thermal radiation and chemical reaction. Mahmoud and Ali [17] investigated the effect of Navier slip imposed on the lower wall on the unsteady hydromagnetic oscillatory flow of an incompressible viscous fluid in a planer channel filled with porous medium. In addition, AbdulHakeem and Sathiyanathan [1] presented analytical solution for two-dimensional oscillatory flow of an incompressible viscous fluid, through a highly porous medium bounded by an infinite vertical plate. Venkateswarlu et al. [26] presented the radiation effects on MHD boundary layer flow of liquid metal over a porous stretching surface in porous medium with heat generation. Jha and Ajibade [8-10] reported some interesting results on the free convective oscillatory flows induced by time dependent boundary conditions in vertical porous plates. While Umavathi et al. [24] studied the unsteady oscillatory flow and heat transfer in a horizontal composite porous medium channel. Venkateswarlu et al. [27-28] presented the heat and mass transfer effects on MHD flows through porous medium over a vertical plate in presence of chemical reaction and thermal radiation. 
All the studies mentioned above ignored the pulsatile nature of the pressure gradient. However, the study of pulsatile flow is of considerable importance in many physiological and engineering problems like the water pumps, fuel pump and pumping of blood through large arteries. Pulsatile flow has attracted several researches in the literature. For example, Sankar and Lee [22] analyzed the pulsatile flow of blood through mild stenosed narrow arteries by treating the blood in the core region as a Casson fluid and the plasma in the peripheral layer as a Newtonian fluid. Massoudi and Phuoc [16] studied the unsteady pulsatile flow of blood in an artery taken the effects of body acceleration into consideration. El-Shahed [6] presented the pulsatile flow of blood through a stenosed porous medium under the influence of body acceleration. Mistra and Pal [18] investigated on the laminar pulsatile flow of blood under the influence of externally imposed body accelerations. Eldabe et al. [7] studied the effect of couple stresses on the MHD of a nonNewtonian unsteady flow between two parallel porous plates. Zuecco and Beg [31] investigated on network numerical simulation applied to pulsatile non-Newtonian flow through a channel with couple stress and wall mass flux effects. Rathod and Tanveer [20] presented the pulsatile flow of couple stress fluid through a porous medium with periodic body acceleration and magnetic field. Recently, Adesanya and Ayeni [2] presented the existence and uniqueness result for couple stress bio fluid flow model by using adomian decomposition method. Adesanya and Makinde [3] investigated analytically on MHD oscillatory slip flow and heat transfer in a channel filled with porous media.

In view of the above studies, the pulsatile slip flow of viscous, incompressible, electrically conducting and hydromagmetic fluid through a parallel channel filled with porous medium is studied. It is assumed that the slip effect depends on shear stress at both walls as applied by Eegunjobi and Makinde [5]. Based on the oscillatory nature of the flow, the problem is formulated, non-dimensionalized and exact solution of the problem is obtained. By means of the presented solutions, the skin friction, heat transfer and mass transfer coefficients of physical importance can be rigorously investigated. The rest of the paper is organized as follows; section two presents the formation of the problem. Section three describes the method of solution and results are discussed in section four, and finally section five provides a conclusion of the paper.

\section{Formation of The Problem}

We consider the unsteady laminar slip flow of an incompressible, viscous and electrically conducting fluid through a channel with non-uniform wall temperature bounded by two parallel plates separated by a distance $a$. The channel is assumed to be filled with a saturated porous medium. A uniform magnetic field of strength $B_{0}$ is applied perpendicular to the plates. The above plate is heated at constant temperature and the thermal radiation effect is also taken in to account. It is assumed that there exist a homogeneous chemical reaction of first order with constant rate $K_{r}^{*}$ between the diffusing species and the fluid. Initially i.e. at time $t \leq 0$, both the fluid and plate are at rest and at uniform temperature $T_{0}$. Also species concentration within the fluid is maintained at uniform concentration $C_{0}$. Geometry of the problem is presented in Fig. 1 . We choose a 
Cartesian coordinate system $(x, y)$ where $x$-lies along the centre of the channel, $y$ - is the distance measured in the normal section such that $y=a$ is the channel's width as shown in the Fig. 1. Under the assumptions made by Adesanya and Makinde [3], as well as of the usual Boussinesq's approximation, the equations of conservation of mass, momentum, energy and concentration governing the free convective nonlinear boundary layer flow over a laminar porous plate in porous medium can be expressed as:

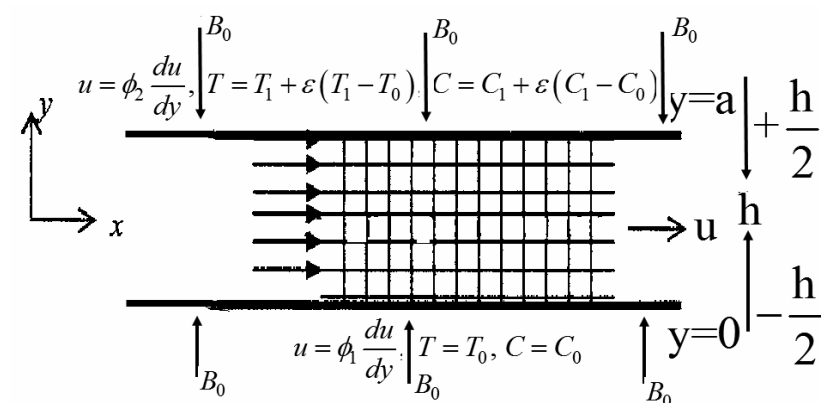

Fig.1: Geometry of the problem

Continuity equation:

$$
\frac{\partial v}{\partial y}=0
$$

Momentum equation:

$$
\frac{\partial u}{\partial t}=-\frac{1}{\rho} \frac{d p}{d x}+v \frac{\partial^{2} u}{\partial y^{2}}+g \beta_{T}\left(T-T_{0}\right)+g \beta_{C}\left(C-C_{0}\right)-\frac{\sigma_{e} B_{0}^{2}}{\rho} u-\frac{v}{K} u
$$

Energy equation:

$$
\frac{\partial T}{\partial t}=\frac{K_{T}}{\rho c_{p}} \frac{\partial^{2} T}{\partial y^{2}}+\frac{4 \alpha^{2}}{\rho c_{p}}\left(T-T_{0}\right)
$$

Diffusion equation;

$$
\frac{\partial C}{\partial t}=D_{m} \frac{\partial^{2} C}{\partial y^{2}}+\frac{D_{m} K_{T}}{T_{m}} \frac{\partial^{2} T}{\partial y^{2}}-K_{r}^{*}\left(C-C_{0}\right)
$$

where $u$-fluid velocity in $x$-direction, $v$-fluid velocity along y-direction, $p$-fluid pressure, $g-$ acceleration due to gravity, $\rho$-fluid density, $\beta_{T}$-coefficient of thermal expansion, $\beta_{C}$-coefficient of concentration volume expansion, $t$-time, $K$-permeability of porous medium, $B_{0}$-magnetic induction, $T$-fluid temperature, $T_{0}$-temperature at the cold wall, $K_{T}$-thermal diffusivity of the fluid, $\mathrm{C}$-species concentration in the fluid, $\mathrm{C}_{0}$-concentration at the cold wall, $\sigma_{\mathrm{e}}$-fluid electrical conductivity, $\mathrm{C}_{\mathrm{p}}$-specific heat at constant pressure, $D_{m}$-chemical molecular diffusivity, $T_{m}$-mean fluid temperature, $v$-kinematic viscosity of the fluid, $\alpha^{2}$-dimensional thermal radiation parameter and $K_{r}^{*}$ - chemical reaction respectively. 
We should in prior warn the reader that our model is not the same as that Adesanya and Makinde [3] in which the Schmidt number, chemical reaction and Soret effects were not taken into account. We should in prior emphasize that our intention is not to reproduce the results of Adesanya and Makinde [3]. In fact, the model that we consider differs considerably from that of Adesanya and Makinde [3] in that we use a better approach in the formulation, introduce a Schmidt number, Soret effect and chemical reaction parameter. Analytical closed form solutions are presented for the momentum, the energy and the concentration equations using some proper change of non dimensional variables and parameters.

Assuming that slipping occurs between the plate and fluid, the corresponding initial and boundary conditions of the system of partial differential equations for the fluid flow problem are given below

$$
\left.\begin{array}{l}
u=\phi_{1} \frac{d u}{d y}, \quad T=T_{0}, \quad C=C_{0} \quad \text { at } \quad y=0 \\
u=\phi_{2} \frac{d u}{d y}, T=T_{1}+\varepsilon\left(T_{1}-T_{o}\right) \exp (\text { int }), C=C_{1}+\varepsilon\left(C_{1}-C_{o}\right) \exp (\text { int }) \text { at } y=a
\end{array}\right\}
$$

where $T_{1}$-fluid temperature at the heated plate, $C_{1}$-species concentration at the heated plate, $\phi_{1}-$ cold wall dimensional slip parameter, $\phi_{2}$-heated wall dimensional slip parameter, $n$-frequency of oscillation and $\varepsilon<<1$ is a very small positive constant.

In order to write the governing equations and the boundary conditions in non-dimensional form, we introduce the following non-dimensional quantities

$$
\left.\begin{array}{l}
\psi=\frac{x}{h}, \eta=\frac{y}{h}, U=\frac{h}{v} u, P=\frac{h^{2}}{\rho v^{2}} p, \gamma=\frac{\phi_{1}}{h}, \sigma=\frac{\phi_{2}}{h}, \\
\omega=\frac{h^{2}}{v} n, \quad \tau=\frac{v}{h^{2}} t, \quad \theta=\frac{T-T_{o}}{T_{1}-T_{o}}, \quad \phi=\frac{C-C_{o}}{C_{1}-C_{o}}
\end{array}\right\}
$$

Equations (2), (3) and (4) reduce to the following non-dimensional form

$$
\begin{aligned}
& \frac{\partial U}{\partial \tau}=-\frac{d P}{d \psi}+\frac{\partial^{2} U}{\partial \eta^{2}}+G_{r} \theta+G_{m} \phi-\left[M+\frac{1}{D a}\right] U \\
& \frac{\partial \theta}{\partial \tau}=\frac{1}{P_{r}} \frac{\partial^{2} \theta}{\partial \eta^{2}}+N \theta \\
& \frac{\partial \phi}{\partial \tau}=\frac{1}{S_{c}} \frac{\partial^{2} \phi}{\partial \eta^{2}}+S_{r} \frac{\partial^{2} \theta}{\partial \eta^{2}}-K_{r} \phi
\end{aligned}
$$

Here $G_{r}=\frac{g \beta_{T}\left(T_{1}-T_{o}\right) h^{3}}{v^{2}}$ is the thermal buoyancy force, $G_{m}=\frac{g \beta_{C}\left(C_{1}-C_{0}\right) h^{3}}{v^{2}}$ is the concentration buoyancy force, $M=\frac{\sigma_{e} B_{o}^{2} h^{2}}{\rho v}$ is the magnetic parameter, $D a=\frac{K}{h^{2}}$ is the permeability 
parameter, $P_{r}=\frac{\rho c_{p} \nu}{K_{T}}$ is the Prandtl number, $N=\frac{4 \alpha^{2} h^{2}}{\rho c_{p} \nu}$ is the thermal radiation parameter, $S_{r}=\frac{D_{m} K_{T}\left(T_{1}-T_{o}\right)}{T_{m} v\left(C_{1}-C_{0}\right)}$ is the Soret number, $S_{c}=\frac{v}{D_{m}}$ is the Schmidt number and $K_{r}=\frac{h^{2}}{v} K_{r}^{*}$ is the chemical reaction parameter respectively.

Corresponding initial and boundary conditions, presented by equation (5), in non-dimensional form, are given by

$$
\left.\begin{array}{l}
U=\gamma \frac{d U}{d \eta}, \quad \theta=0, \quad \phi=0 \text { at } \eta=0 \\
U=\sigma \frac{d U}{d \eta}, \theta=1+\varepsilon \exp (i \omega \tau), \phi=1+\varepsilon \exp (i \omega \tau) \text { at } \eta=1
\end{array}\right\}
$$

Following Adesanya and Makinde [3], for purely an oscillatory flow we take the pressure gradient of the form

$\lambda=-\frac{d P}{d \psi}=\lambda_{0}+\varepsilon \lambda_{1} \exp (i \omega t)$

where $\lambda_{0}-$ and $\lambda_{1}-$ are constants and $\omega$ is the frequency of oscillation.

It is now important to calculate physical quantities of primary interest, which are the local wall shear stress or skin friction coefficient, the local surface heat flux and the local surface mass flux. Given the velocity, temperature and concentration fields in the boundary layer, the shear stress $\tau_{w}$, the heat flux $q_{w} q_{w}$ and mass flux $j_{w}$ are obtained by

$$
\begin{aligned}
& \tau_{w}=\mu\left[\frac{\partial u}{\partial y}\right] \\
& q_{w}=-K_{T}\left[\frac{\partial T}{\partial y}\right] \\
& j_{w}=-D_{m}\left[\frac{\partial C}{\partial y}\right]
\end{aligned}
$$

In non-dimensional form the skin-friction coefficient $C_{f}$, heat transfer coefficient $\mathrm{Nu}$ and mass transfer coefficient $S h$ are defined as

$$
\begin{aligned}
& C f=\frac{\tau_{w}}{\rho(v / h)^{2}} \\
& N u=\frac{h q_{w}}{K_{T}\left(T_{1}-T_{o}\right)}
\end{aligned}
$$




$$
S h=\frac{h j_{w}}{D_{m}\left(C_{1}-C_{o}\right)}
$$

Using non-dimensional variables in equation (6) and equations (12) to (14) into equations (15) to (17), we obtain the physical parameters

$$
\begin{aligned}
& C f=\left[\frac{\partial U}{\partial \eta}\right] \\
& N u=-\left[\frac{\partial \theta}{\partial \eta}\right] \\
& S h=-\left[\frac{\partial \phi}{\partial \eta}\right]
\end{aligned}
$$

\section{Solution of the Problem}

Equations (7) to (9) are coupled non-linear partial differential equations and these cannot be solved in closed form. So, we reduce these non-linear partial differential equations into a set of ordinary differential equations, which can be solved analytically. This can be done by assuming the trial solutions for the velocity, temperature and concentration of the fluid as

$$
\begin{aligned}
& U(\eta, \tau)=U_{0}(\eta)+\varepsilon \exp (i \omega \tau) U_{1}(\eta)+o\left(\varepsilon^{2}\right) \\
& \theta(\eta, \tau)=\theta_{0}(\eta)+\varepsilon \exp (i \omega \tau) \theta_{1}(\eta)+o\left(\varepsilon^{2}\right) \\
& \phi(\eta, \tau)=\phi_{0}(\eta)+\varepsilon \exp (i \omega \tau) \phi_{1}(\eta)+o\left(\varepsilon^{2}\right)
\end{aligned}
$$

Substituting equations (21) to (23) into equations (7) to (9), then equating the harmonic and nonharmonic terms and neglecting the higher order terms of $0\left(\varepsilon^{2}\right)$, we obtain

$$
\begin{aligned}
& U_{0}^{\prime \prime}-\left[M+\frac{1}{D a}\right] U_{0}=-\left[G_{r} \theta_{0}+G_{m} \phi_{0}+\lambda_{0}\right] \\
& U_{1}^{\prime \prime}-\left[M+\frac{1}{D a}+i \omega\right] U_{1}=-\left[G_{r} \theta_{1}+G_{m} \phi_{1}+\lambda_{1}\right] \\
& \theta_{0}^{\prime \prime}+N P_{r} \theta_{0}=0 \\
& \theta_{1}^{\prime \prime}+P_{r}(N-i \omega) \theta_{1}=0 \\
& \phi_{0}^{\prime \prime}-S_{c} K_{r} \phi_{0}=-S_{c} S_{r} \theta_{o}^{\prime \prime} \\
& \phi_{1}^{\prime \prime}-S_{c}\left(K_{r}+i \omega\right) \phi_{1}=-S_{c} S_{r} \theta_{1}^{\prime \prime}
\end{aligned}
$$

where the prime denotes the ordinary differentiation with respect to $\eta$. 
Corresponding initial and boundary conditions, presented by equation (10), can be written as

$$
\left.\begin{array}{lllll}
U_{o}=\gamma \frac{d U_{o}}{d \eta}, & U_{1}=\gamma \frac{d U_{1}}{d \eta}, \quad \theta_{o}=0, \theta_{1}=0, & \phi_{o}=0, \quad \phi_{1}=0 & \text { at } & \eta=0 \\
U_{o}=\sigma \frac{d U_{o}}{d \eta}, & U_{1}=\sigma \frac{d U_{1}}{d \eta}, & \theta_{o}=1, \theta_{1}=1, \quad \phi_{o}=1, \quad \phi_{1}=1 & \text { at } & \eta=1
\end{array}\right\}
$$

The analytical solutions of equations (24) to (29) with the boundary conditions in equation (30), are given by

$$
\begin{aligned}
& U_{o}=A_{22} \exp \left(-m_{5} \eta\right)+A_{21} \exp \left(m_{5} \eta\right)+A_{8}+\frac{A_{6} \sin \left(m_{1} \eta\right)}{\sin \left(m_{1}\right)}-\frac{A_{7} \sinh \left(m_{3} \eta\right)}{\sinh \left(m_{3}\right)} \\
& U_{1}=A_{39} \exp \left(-m_{6} \eta\right)+A_{38} \exp \left(m_{6} \eta\right)+A_{25}+\frac{A_{23} \sin \left(m_{2} \eta\right)}{\sin \left(m_{2}\right)}-\frac{A_{24} \sinh \left(m_{4} \eta\right)}{\sinh \left(m_{4}\right)} \\
& \theta_{o}=\frac{\sin \left(m_{1} \eta\right)}{\sin \left(m_{1}\right)} \\
& \theta_{1}=\frac{\sin \left(m_{2} \eta\right)}{\sin \left(m_{2}\right)} \\
& \phi_{o}=\frac{A_{3} \sinh \left(m_{3} \eta\right)}{\sinh \left(m_{3}\right)}-\frac{A_{2} \sin \left(m_{1} \eta\right)}{\sin \left(m_{1}\right)} \\
& \phi_{1}=\frac{A_{5} \sinh \left(m_{4} \eta\right)}{\sinh \left(m_{4}\right)}-\frac{A_{4} \sin \left(m_{2} \eta\right)}{\sin \left(m_{2}\right)}
\end{aligned}
$$

By substituting equations (31) to (36) into equations (21) to (23) we obtained solutions for the fluid velocity, temperature and concentration and are presented in the following form

$$
\begin{aligned}
& U(\eta, \tau)=\left[A_{22} \exp \left(-m_{5} \eta\right)+A_{21} \exp \left(m_{5} \eta\right)+A_{8}+\frac{A_{6} \sin \left(m_{1} \eta\right)}{\sin \left(m_{1}\right)}-\frac{A_{7} \sinh \left(m_{3} \eta\right)}{\sinh \left(m_{3}\right)}\right]+ \\
& \varepsilon \exp (i \omega \tau)\left[A_{39} \exp \left(-m_{6} \eta\right)+A_{38} \exp \left(m_{6} \eta\right)+A_{25}+\frac{A_{23} \sin \left(m_{2} \eta\right)}{\sin \left(m_{2}\right)}-\frac{A_{24} \sinh \left(m_{4} \eta\right)}{\sinh \left(m_{4}\right)}\right] \\
& \theta(\eta, \tau)=\frac{\sin \left(m_{1} \eta\right)}{\sin \left(m_{1}\right)}+\varepsilon \exp (i \omega \tau)\left[\frac{\sin \left(m_{2} \eta\right)}{\sin \left(m_{2}\right)}\right] \\
& \phi(\eta, \tau)=\left[\frac{A_{3} \sinh \left(m_{3} \eta\right)}{\sinh \left(m_{3}\right)}-\frac{A_{2} \sin \left(m_{1} \eta\right)}{\sin \left(m_{1}\right)}\right]+\varepsilon \exp (i \omega \tau)\left[\frac{A_{5} \sinh \left(m_{4} \eta\right)}{\sinh \left(m_{4}\right)}-\frac{A_{4} \sin \left(m_{2} \eta\right)}{\sin \left(m_{2}\right)}\right]
\end{aligned}
$$

\subsection{Skin friction}

From the velocity field, the skin friction at the plate can be obtained, which in non-dimensional form is given by

$$
\begin{aligned}
& C f=\left[A_{21} m_{5} \exp \left(m_{5} \eta\right)-A_{22} m_{5} \exp \left(-m_{5} \eta\right)+\frac{A_{6} m_{1} \cos \left(m_{1} \eta\right)}{\sin \left(m_{1}\right)}-\frac{A_{7} m_{3} \cosh \left(m_{3} \eta\right)}{\sinh \left(m_{3}\right)}\right]+ \\
& \varepsilon \exp (i \omega \tau)\left[A_{38} m_{6} \exp \left(m_{6} \eta\right)-A_{39} m_{6} \exp \left(-m_{6} \eta\right)+\frac{A_{23} m_{2} \cos \left(m_{2} \eta\right)}{\sin \left(m_{2}\right)}-\frac{A_{24} m_{4} \cosh \left(m_{4} \eta\right)}{\sinh \left(m_{4}\right)}\right]
\end{aligned}
$$




\subsection{Nusselt number}

From temperature field, we obtained heat transfer coefficient which is given in non-dimensional form as

$$
N u=-\frac{m_{1} \cos \left(m_{1} \eta\right)}{\sin \left(m_{1}\right)}-\varepsilon \exp (i \omega \tau)\left[\frac{m_{2} \cos \left(m_{2} \eta\right)}{\sin \left(m_{2}\right)}\right]
$$

\subsection{Sherwood number}

From concentration field, we obtained mass transfer coefficient which is given in non-dimensional form as

$S h=\left[\frac{A_{2} m_{1} \cos \left(m_{1} \eta\right)}{\sin \left(m_{1}\right)}-\frac{A_{3} m_{3} \cosh \left(m_{3} \eta\right)}{\sinh \left(m_{3}\right)}\right]+\varepsilon \exp (i \omega \tau)\left[\frac{A_{4} m_{2} \cos \left(m_{2} \eta\right)}{\sin \left(m_{2}\right)}-\frac{A_{5} m_{4} \cosh \left(m_{4} \eta\right)}{\sinh \left(m_{4}\right)}\right]$

\section{Results and Discussion}

In order to investigate the influence of various physical parameters such as mass diffusion parameter $S_{c}$, Soret effect $S_{r}$, chemical reaction parameter $K_{r}$, radiation parameter $N$, Prandtl number $P_{r}$, pressure gradient $\lambda$, magnetic parameter $M$, Darcy parameter $D a$, thermal Grashof number $G_{r}$, solutal Grashof number $G_{m}$, cold wall slip parameter $\gamma$, heated wall slip parameter $\sigma$ and time $\tau$ on the flow-field, fluid velocity $U$, temperature $\theta$ and concentration $\phi$ have been studied analytically and computed results of the analytical solutions from equations (37) to (39) are displayed graphically from Figs. 2 to 23 for various values of these physical parameters. The numerical values of skin friction, Nusselt number and Sherwood number computed from analytical solutions, presented by equations (40) to (42) are presented in tabular form in tables 1 to 6 for various values of different physical parameters. In the present study following default parameter values are adopted for computations: $S_{c}=0.60, S_{r}=1.0, K_{r}=0.5, N=2.0, P_{r}=0.71, \lambda=1, M=$ $1.0, D a=0.5, \tau=1.0, G_{r}=5.0, G_{m}=2.0, \gamma=0.2, \sigma=0.4, \omega=0.1$ and $\varepsilon=0.01$. Therefore all the graphs and tables are corresponding to these values unless specifically indicated on the appropriate graph or table.

Figs. 2 to 4 , shows the plot of fluid velocity $U$, temperature $\theta$ and concentration $\phi$ of the flow field against different values of Prandtl number $P_{r}$ taking other parameters are constant. The Prandtl number defines the ratio of momentum diffusivity to thermal diffusivity. It is evident from Figs. 2 to 4 , velocity $U$ and temperature $\theta$ increases on increasing Prandtl number $P_{r}$ whereas concentration $\phi$ decreases on increasing $\mathrm{P}_{\mathrm{r}}$ throughout the boundary layer region.

It is observed that from Figs. 5 to 7, both the velocity $U$ and temperature $\theta$ increases on increasing the radiation parameter $N$ whereas concentration $\phi$ decreases on increasing radiation parameter $N$ throughout the boundary layer region. It is noted from Figs. 8 to 10 that the fluid velocity $U$, temperature $\theta$ and concentration $\phi$ attain their steady state for large time $\tau$. 


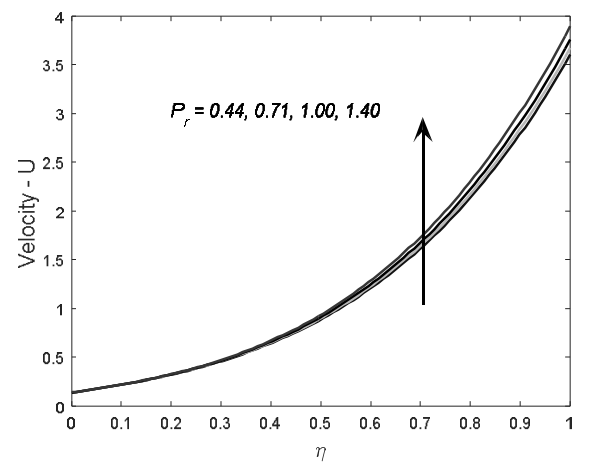

Fig.2: Influence of Prandtl number on velocity profiles.

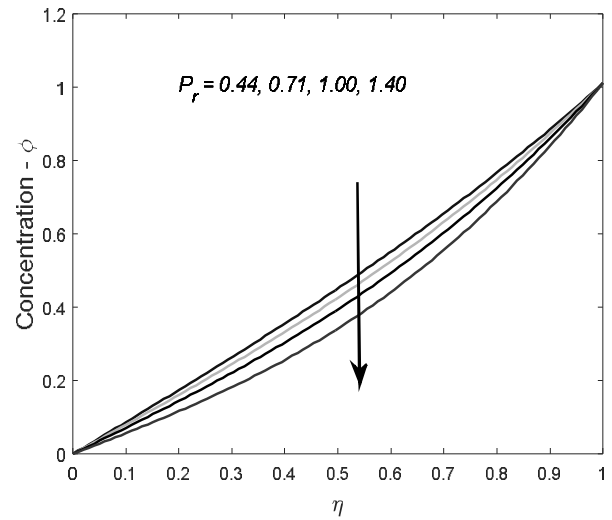

Fig.4: Influence of Prandtl number on concentration profiles.

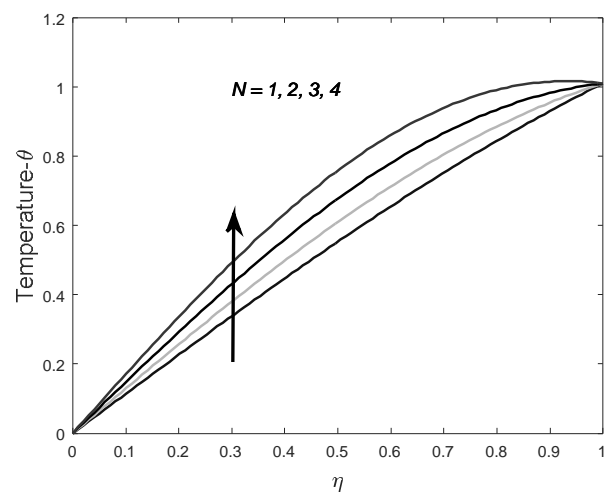

Fig.6: Influence of radiation parameter on temperature profiles.

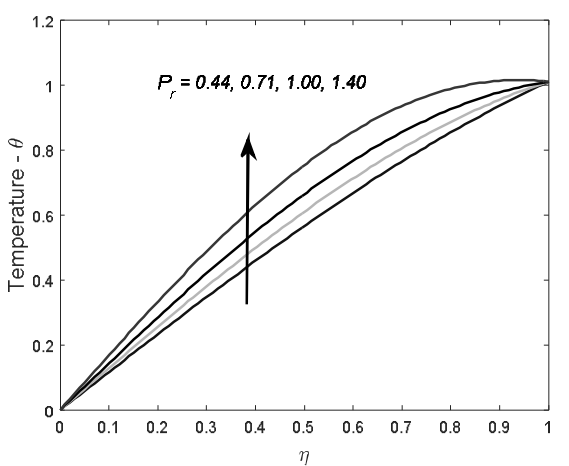

Fig.3: Influence of Prandtl number on temperature profiles.

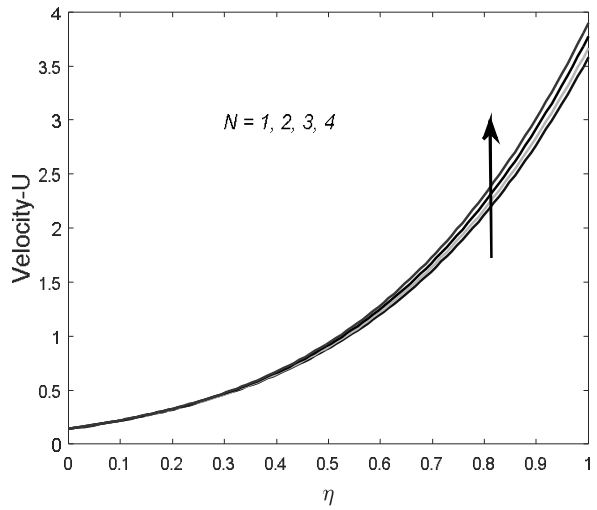

Fig.5: Influence of radiation parameter on velocity profiles.

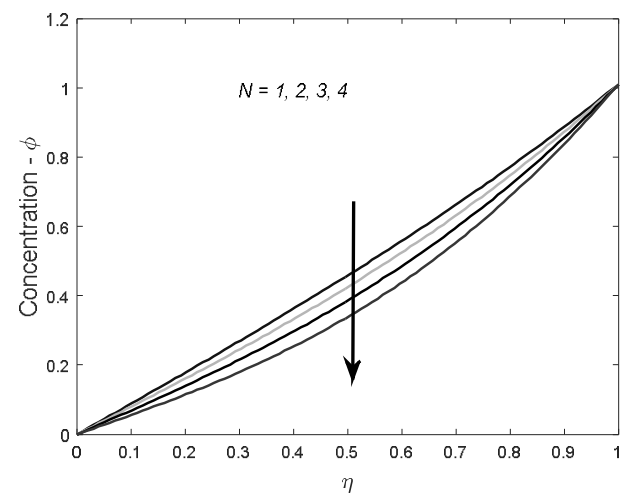

Fig.7: Influence of radiation parameter on concentration profiles. 


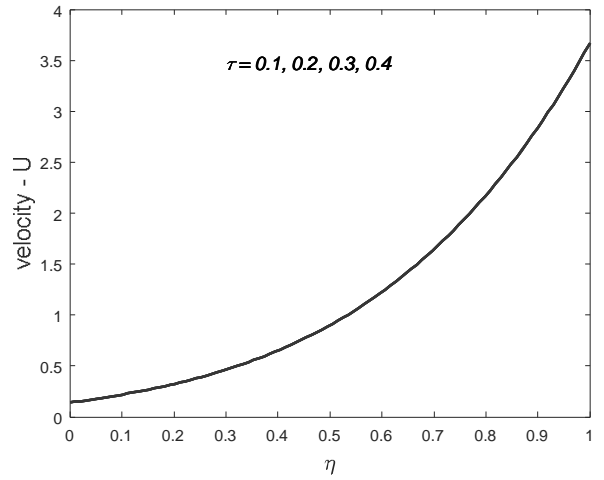

Fig.8: Influence of time on velocity profiles.

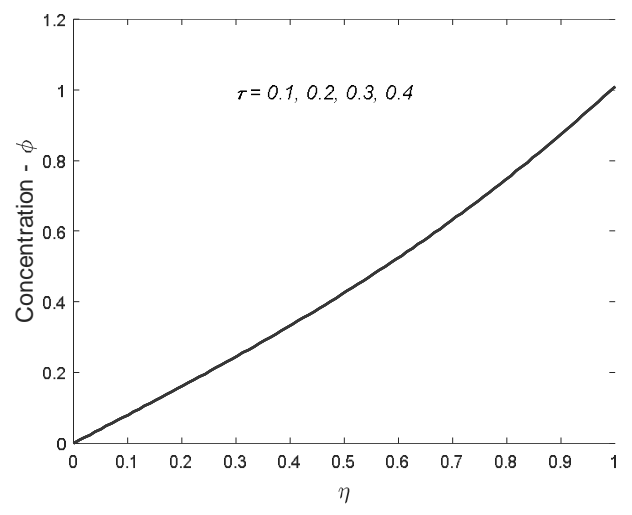

Fig.10: Influence of time on concentration profiles.

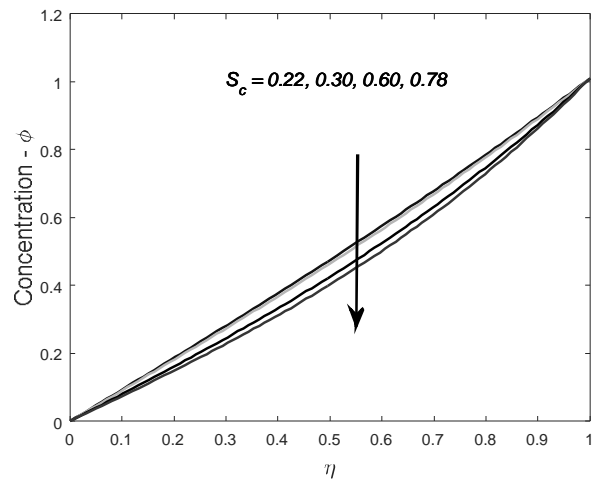

Fig.12: Influence of Schmidt number on concentration profiles.

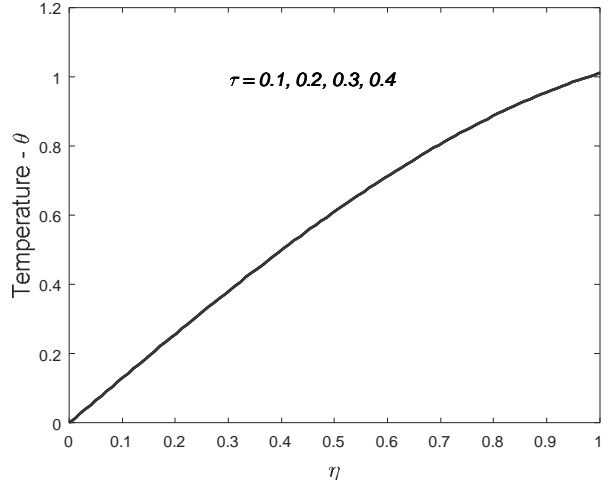

Fig.9: Influence of time on temperature profiles.

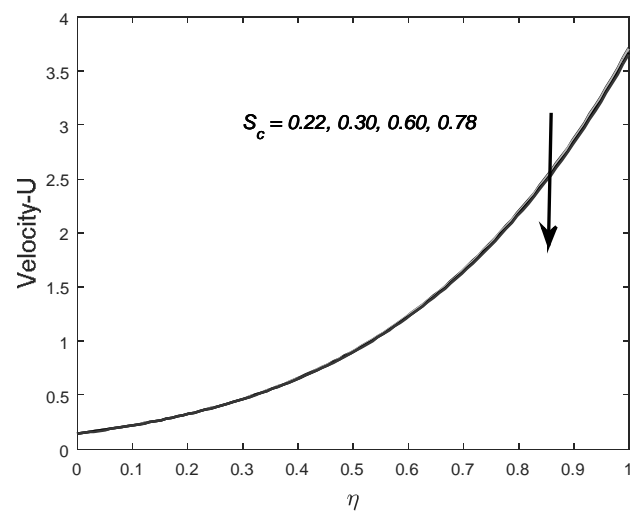

Fig.11: Influence of Schmidt number on velocity profiles.

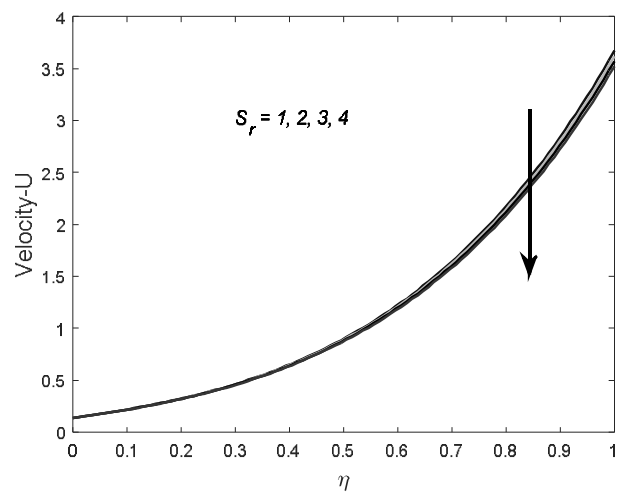

Fig.13: Influence of Soret number on velocity profiles. 


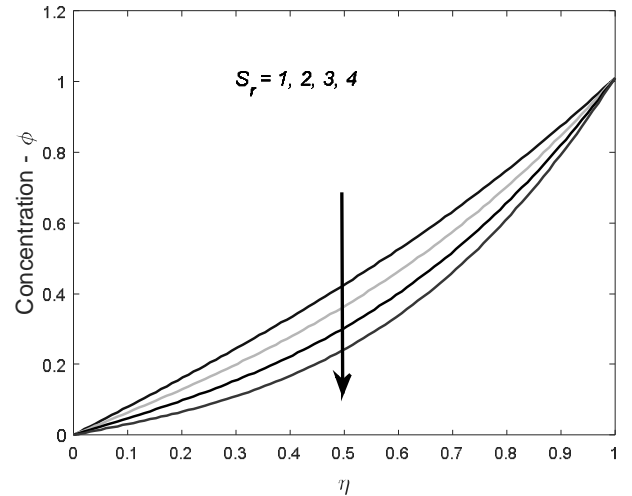

Fig.14: Influence of Soret number on concentration profiles.

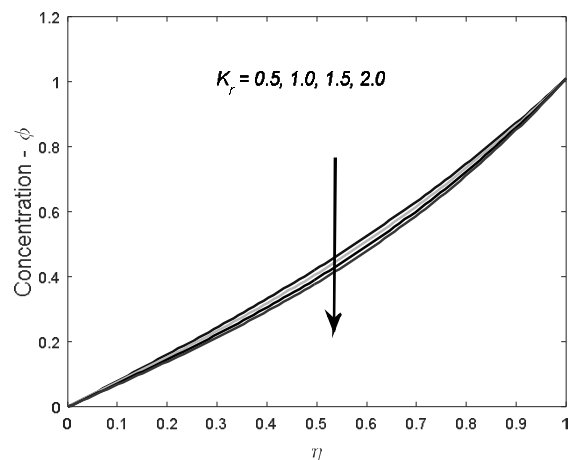

Fig.16: Influence of chemical reaction parameter on concentration profiles.

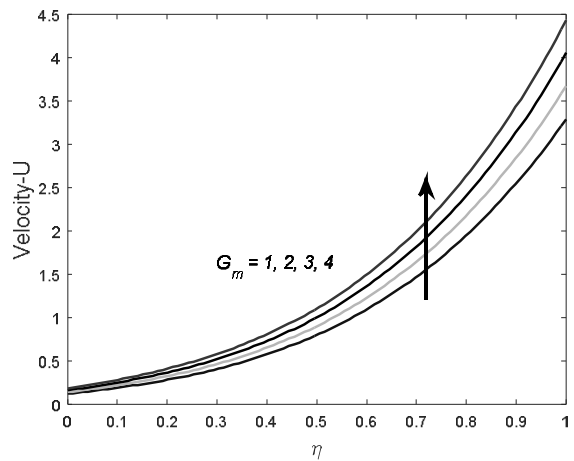

Fig.18: Influence of solutal Grashof number on velocity profiles.

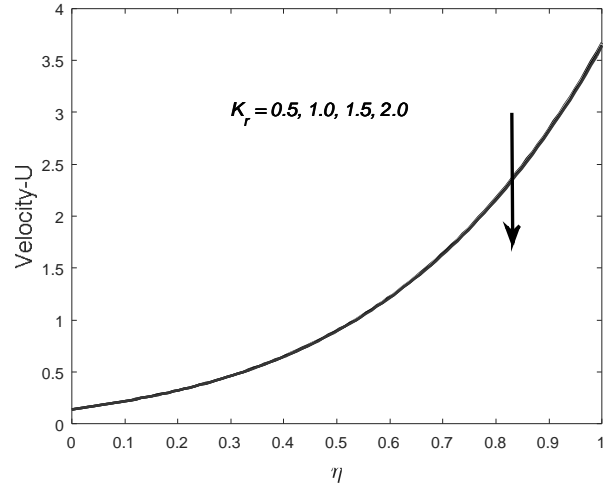

Fig.15: Influence of chemical reaction parameter on velocity profiles.

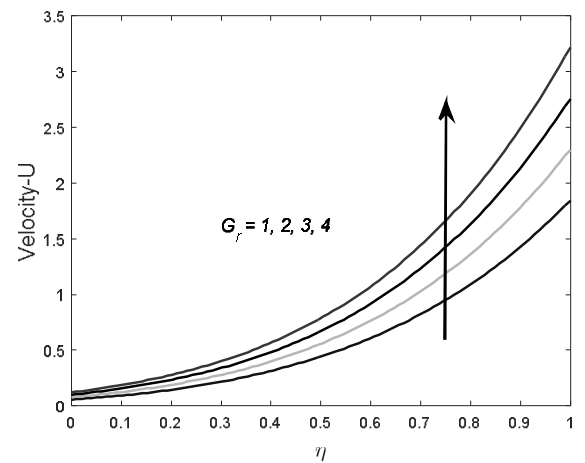

Fig.17: Influence of Grashof number on velocity profiles.

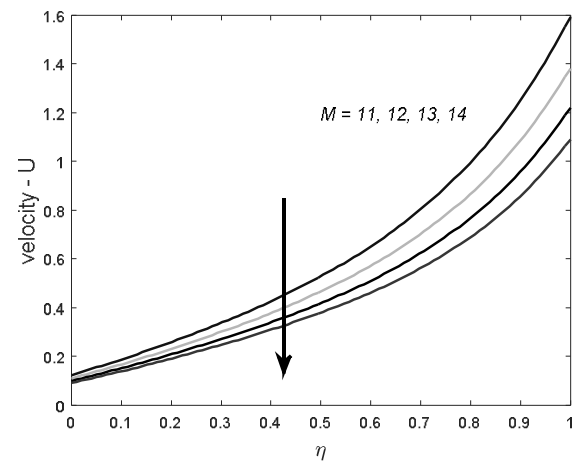

Fig.19: Influence of magnetic parameter on velocity profiles. 


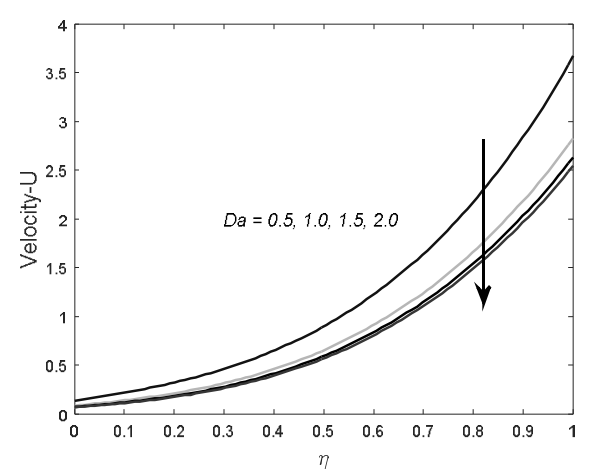

Fig.20: Influence of Darcy parameter on velocity profiles.

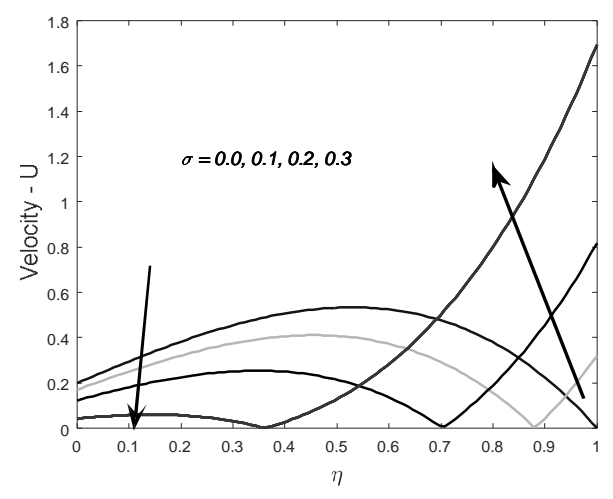

Fig.22: Influence of heated wall slip parameter on velocity profiles.

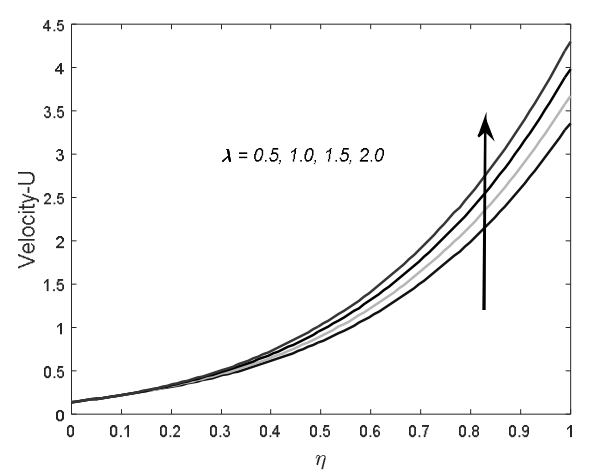

Fig.21: Influence of pressure gradient on velocity profiles.

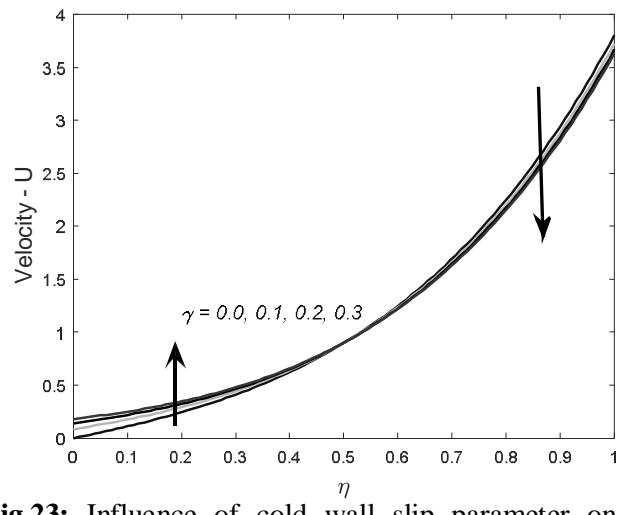

Fig.23: Influence of cold wall slip parameter on velocity profiles.

The nature of fluid velocity $U$ and concentration $\phi$ in presence of foreign species such as Hydrogen $\left(S_{c}=0.22\right)$, Helium $\left(S_{c}=0.30\right)$, Water vapour $\left(S_{c}=0.60\right)$, Ammonia $\left(S_{c}=0.78\right)$ is shown in Figs, 11 and 12. Physically, Schmidt number $S_{c}$ signifies the relative strength of viscosity to chemical molecular diffusivity. It is observed that $U$ and $\phi$ decreases on increasing $S_{c}$ throughout the boundary layer region. The flow field suffers a decrease in velocity $U$ and concentration $\phi$ in presence of heavier diffusing species.

Figs. 13 and 14 depict effects of Soret number $S_{r}$ on the velocity and species concentration distribution of the flow field. It is noticed that, velocity $U$ and species concentration $\phi$ is found to decreases on increasing Soret number $S_{r}$ throughout the boundary layer region.

Figs. 15 and 16 demonstrate the effects of chemical reaction parameter $K_{r}$ on the velocity and species concentration. It is observed that, both velocity $U$ and species concentration $\phi$ decreases on increasing the chemical reaction parameter $K_{r}$. This implies that, chemical reaction tends to reduce the velocity and species concentration. 
The effect of Grashof number $G_{r}$ for heat transfer on the velocity $\mathrm{U}$ of the flow field is presented in Fig. 17. Physically, Grashof number for heat transfer $G_{r}$ signifies the relative strength of thermal buoyancy force to viscous hydrodynamic force in the boundary layer. A study of the curves shows that Grashof number $G_{r}$ for heat transfer accelerates the velocity $U$ of the flow field at all points. This is due to the reason that there is an enhancement in thermal buoyancy force. The effect of Grashof number $G_{m}$ for mass transfer on the velocity $U$ of the flow field is presented in Fig. 18 . Physically, Grashof number $G_{m}$ for mass transfer signifies the relative strength of species buoyancy force to viscous hydrodynamic force in the boundary layer. A study of the curves shows that the Grashof number $G_{m}$ for mass transfer accelerates the velocity $U$ of the flow field at all points. This is due to the reason that there is an enhancement in concentration buoyancy force.

Fig. 19 depicts the influence of magnetic field $M$ on the velocity $U$ of the flow field. It is noticed from Fig. 19 that, $U$ decreases on increasing the magnetic parameter $M$ throughout the boundary layer region. This is due to the fact that application of a magnetic field to an electrically conducting fluid gives rise a mechanical force, called Lorentz force, which has a tendency to resist fluid motion in the flow field. Fig. 20 demonstrates the influence of Darcy parameter $D a$ on fluid velocity U. It is evident from Fig. 20, fluid velocity $U$ decreases on increasing Da throughout the boundary layer region. It is observed from Fig. 21 that, fluid velocity $U$ increases on increasing pressure gradient $\lambda$.

Table 1: Skin friction coefficient values when $N=2, P_{r}=0.71, S_{c}=0.6, \lambda=1, \tau=1, S_{r}=1, K_{r}=0.5, \gamma=$ $0.2, \sigma=0.4, \omega=0.1, \varepsilon=0.01$.

\begin{tabular}{|c|c|c|c|c|c|}
\hline$G_{r}$ & $G_{m}$ & $M$ & $D a$ & \multicolumn{2}{|c|}{ Skin friction $C_{f}$} \\
\cline { 5 - 6 } & & & & Cold wall & Heated wall \\
\hline 1.0 & 2.0 & 1.0 & 0.5 & 0.2916 & 4.6044 \\
\hline 2.0 & 2.0 & 1.0 & 0.5 & 0.3944 & 5.7470 \\
\hline 3.0 & 2.0 & 1.0 & 0.5 & 0.4971 & 6.8897 \\
\hline 4.0 & 2.0 & 1.0 & 0.5 & 0.5999 & 8.0323 \\
\hline 5.0 & 1.0 & 1.0 & 0.5 & 0.6033 & 8.2235 \\
\hline 5.0 & 2.0 & 1.0 & 0.5 & 0.7026 & 17750 \\
\hline 5.0 & 3.0 & 1.0 & 0.5 & 0.8019 & 11.0780 \\
\hline 5.0 & 4.0 & 1.0 & 0.5 & 0.9013 & 3.9783 \\
\hline 5.0 & 2.0 & 11.0 & 0.5 & 0.6168 & 3.4509 \\
\hline 5.0 & 2.0 & 12.0 & 0.5 & 0.5508 & 3.0442 \\
\hline 5.0 & 2.0 & 13.0 & 0.5 & 0.4987 & 2.7212 \\
\hline 5.0 & 2.0 & 14.0 & 0.5 & 0.4565 & 9.1750 \\
\hline 5.0 & 2.0 & 1.0 & 0.5 & 0.7026 & 6.0742 \\
\hline 5.0 & 2.0 & 1.0 & 1.0 & 0.4455 & 6.3634 \\
\hline 5.0 & 2.0 & 1.0 & 1.5 & 0.3821 & 0.3531 \\
\hline 5.0 & 2.0 & 1.0 & 2.0 & & \\
\hline
\end{tabular}

The nature of fluid velocity $U$ on the heated wall slip parameter $\sigma$ and cold wall slip parameter $\gamma$ is shown in Figs 22 and 23. It is observed that, $U$ decreases in a region near to the plate and increases 
in the region away from the plate on increasing heated wall slip parameter $\sigma$ whereas $U$ increases in a region near to the plate and it decreases in the region away from the plate on increasing cold wall slip parameter $\gamma$. An increase in the heated wall slip parameter $\sigma$ decreases the fluid velocity minimally at the cold wall and increasing the heated wall slip parameter causes a flow reversal towards the heated wall. It is observed that $\sigma=0$ corresponds to the pulsatile case with no slip condition at the heated wall in Fig 22.

Table 2: Skin friction coefficient values when $G_{m}=2, G_{r}=5, M=1, \lambda=1, \tau=1, D a=0.5, K_{r}=0.5, \gamma=$ $0.2, \sigma=0.4, \omega=0.1, \varepsilon=0.01$.

\begin{tabular}{|c|c|c|c|c|c|}
\hline$P_{r}$ & $N$ & $S_{c}$ & $S_{r}$ & \multicolumn{2}{|c|}{ Skin friction $C_{f}$} \\
\cline { 5 - 6 } & & & & Cold wall & Heated wall \\
\hline 0.44 & 2.0 & 0.60 & 1.0 & 0.6999 & 9.0021 \\
\hline 0.71 & 2.0 & 0.60 & 1.0 & 0.7026 & 9.1750 \\
\hline 1.00 & 2.0 & 0.60 & 1.0 & 0.7055 & 9.3857 \\
\hline 1.40 & 2.0 & 0.60 & 1.0 & 0.7094 & 9.7301 \\
\hline 0.71 & 1.0 & 0.60 & 1.0 & 0.6990 & 9.9517 \\
\hline 0.71 & 2.0 & 0.60 & 1.0 & 0.7026 & 9.4370 \\
\hline 0.71 & 3.0 & 0.60 & 1.0 & 0.7062 & 9.7493 \\
\hline 0.71 & 4.0 & 0.60 & 1.0 & 0.7096 & 9.2786 \\
\hline 0.71 & 2.0 & 0.22 & 1.0 & 0.7046 & 9.2564 \\
\hline 0.71 & 2.0 & 0.30 & 1.0 & 0.7042 & 9.1750 \\
\hline 0.71 & 2.0 & 0.60 & 1.0 & 0.7026 & 9.1272 \\
\hline 0.71 & 2.0 & 0.78 & 1.0 & 0.7017 & 9.1750 \\
\hline 0.71 & 2.0 & 0.60 & 1.0 & 0.7026 & 8.9217 \\
\hline 0.71 & 2.0 & 0.60 & 2.0 & 0.7003 & 8.7951 \\
\hline 0.71 & 2.0 & 0.60 & 3.0 & 0.6981 & \\
\hline 0.71 & 2.0 & 0.60 & 4.0 & 0.6958 & \\
\hline
\end{tabular}

Table 3: Skin friction coefficient values when $G_{m}=2, G_{r}=5, M=1, \lambda=1, \tau=1, D a=0.5, K_{r}=0.5, \gamma=0.2$, $\sigma=0.4, \omega=0.1, \varepsilon=0.01$.

\begin{tabular}{|c|c|c|c|c|c|c|}
\hline$K_{r}$ & $\lambda$ & $\sigma$ & $\gamma$ & $\tau$ & \multicolumn{2}{|c|}{ Skin friction $C_{f}$} \\
\cline { 6 - 7 } & & & & & Cold wall & Heated wall \\
\hline 0.5 & 1.0 & 0.4 & 0.2 & 1.0 & 0.7026 & 9.1750 \\
\hline 1.0 & 1.0 & 0.4 & 0.2 & 1.0 & 0.7018 & 9.1420 \\
\hline 1.5 & 1.0 & 0.4 & 0.2 & 1.0 & 0.7010 & 9.1106 \\
\hline 2.0 & 1.0 & 0.4 & 0.2 & 1.0 & 0.7002 & 9.0808 \\
\hline 0.5 & 0.5 & 0.4 & 0.2 & 1.0 & 0.7075 & 8.3957 \\
\hline 0.5 & 1.0 & 0.4 & 0.2 & 1.0 & 0.7026 & 9.1750 \\
\hline 0.5 & 1.5 & 0.4 & 0.2 & 1.0 & 0.6978 & 9.9543 \\
\hline 0.5 & 2.0 & 0.4 & 0.2 & 1.0 & 0.6929 & 10.7337 \\
\hline 0.5 & 1.0 & 0.0 & 0.2 & 1.0 & 0.9937 & 2.6223 \\
\hline 0.5 & 1.0 & 0.1 & 0.2 & 1.0 & 0.8462 & 3.1923 \\
\hline 0.5 & 1.0 & 0.2 & 0.2 & 1.0 & 0.6167 & 4.0789 \\
\hline 0.5 & 1.0 & 0.3 & 0.2 & 1.0 & 0.2107 & 5.6472 \\
\hline
\end{tabular}




\begin{tabular}{|l|l|l|l|l|l|l|}
\hline 0.5 & 1.0 & 0.4 & 0.0 & 1.0 & 1.0475 & 9.5138 \\
\hline 0.5 & 1.0 & 0.4 & 0.1 & 1.0 & 0.8411 & 9.3110 \\
\hline 0.5 & 1.0 & 0.4 & 0.2 & 1.0 & 0.7026 & 9.1750 \\
\hline 0.5 & 1.0 & 0.4 & 0.3 & 1.0 & 0.6033 & 9.0774 \\
\hline 0.5 & 1.0 & 0.4 & 0.2 & 0.1 & 0.7027 & 9.1757 \\
\hline 0.5 & 1.0 & 0.4 & 0.2 & 0.2 & 0.7027 & 9.1757 \\
\hline 0.5 & 1.0 & 0.4 & 0.2 & 0.3 & 0.7027 & 9.1757 \\
\hline 0.5 & 1.0 & 0.4 & 0.2 & 0.4 & 0.7027 & 9.1757 \\
\hline
\end{tabular}

Table 4: Nusselt number values when $\omega=0.1, \varepsilon=0.01$.

\begin{tabular}{|c|c|c|c|c|}
\hline \multirow{2}{*}{$P_{r}$} & $N$ & \multirow{2}{*}{$\tau$} & \multicolumn{2}{|c|}{ Nusselt number $N u$} \\
\cline { 3 - 5 } & & & Cold wall & Heated wall \\
\hline 0.44 & 2.0 & 1.0 & -1.1748 & -0.6947 \\
\hline 0.71 & 2.0 & 1.0 & -1.2955 & -0.4795 \\
\hline 1.00 & 2.0 & 1.0 & -1.4460 & -0.2255 \\
\hline 1.40 & 2.0 & 1.0 & -1.6989 & -0.1739 \\
\hline 0.71 & 1.0 & 1.0 & -1.1402 & -0.7588 \\
\hline 0.71 & 2.0 & 1.0 & -1.2955 & -0.4795 \\
\hline 0.71 & 3.0 & 1.0 & -1.4832 & -0.1648 \\
\hline 0.71 & 4.0 & 1.0 & -1.7132 & -0.1957 \\
\hline 0.71 & 2.0 & 0.1 & -1.2956 & -0.4795 \\
\hline 0.71 & 2.0 & 0.2 & -1.2956 & -0.4795 \\
\hline 0.71 & 2.0 & 0.3 & -1.2956 & -0.4795 \\
\hline 0.71 & 2.0 & 0.4 & -1.2956 & -0.4795 \\
\hline
\end{tabular}

Table 5: Sherwood number values when $S_{r}=0.6, K_{r}=0.5, \tau=1, \omega=0.1, \varepsilon=0.01$.

\begin{tabular}{|c|c|c|c|c|}
\hline \multirow{2}{*}{$P_{r}$} & $N$ & $S_{c}$ & \multicolumn{2}{|c|}{ Sherwood number $S h$} \\
\cline { 4 - 5 } & & 0.60 & 0.8656 & Heated wall \\
\hline 0.44 & 2.0 & 0.60 & 0.7956 & 1.2943 \\
\hline 0.71 & 2.0 & 0.60 & 0.7082 & 1.4208 \\
\hline 1.00 & 2.0 & 0.60 & 0.5614 & 1.5699 \\
\hline 1.40 & 2.0 & 0.60 & 0.8857 & 1.8042 \\
\hline 0.71 & 1.0 & 0.60 & 0.7956 & 1.2567 \\
\hline 0.71 & 2.0 & 0.60 & 0.6866 & 1.4208 \\
\hline 0.71 & 3.0 & 0.60 & 0.5530 & 1.6056 \\
\hline 0.71 & 4.0 & 0.22 & 0.9296 & 1.8170 \\
\hline 0.71 & 2.0 & 0.30 & 0.9009 & 1.1625 \\
\hline 0.71 & 2.0 & 0.60 & 0.7956 & 1.2174 \\
\hline 0.71 & 2.0 & 0.78 & 0.7340 & 1.4208 \\
\hline 0.71 & 2.0 & & & 1.5409 \\
\hline
\end{tabular}


Table 6: Sherwood number values when $P_{r}=0.71, S_{c}=0.6, N=2, \omega=0.1, \varepsilon=0.01$.

\begin{tabular}{|c|c|c|c|c|}
\hline \multirow[t]{2}{*}{$S_{r}$} & \multirow[t]{2}{*}{$K_{r}$} & \multirow[t]{2}{*}{$\tau$} & \multicolumn{2}{|c|}{ Sherwood number $S h$} \\
\hline & & & Cold wall & Heated wall \\
\hline 1.0 & 0.5 & 1.0 & 0.7956 & 1.4208 \\
\hline 2.0 & 0.5 & 1.0 & 0.6299 & 1.7326 \\
\hline 3.0 & 0.5 & 1.0 & 0.4643 & 2.0444 \\
\hline 4.0 & 0.5 & 1.0 & 0.2987 & 2.3562 \\
\hline 1.0 & 0.5 & 1.0 & 0.7956 & 1.4208 \\
\hline 1.0 & 1.0 & 1.0 & 0.7554 & 1.5100 \\
\hline 1.0 & 1.5 & 1.0 & 0.7178 & 1.5961 \\
\hline 1.0 & 2.0 & 1.0 & 0.6827 & 1.6793 \\
\hline 1.0 & 0.5 & 0.1 & 0.7956 & 1.4208 \\
\hline 1.0 & 0.5 & 0.2 & 0.7956 & 1.4208 \\
\hline 1.0 & 0.5 & 0.3 & 0.7956 & 1.4208 \\
\hline 1.0 & 0.5 & 0.4 & 0.7956 & 1.4208 \\
\hline
\end{tabular}

The numerical values of skin friction coefficient $C_{f}$, obtained from the exact analytical solution, presented by equation (40), are presented in tabular form in tables 1 to 3. It is clear that, the skin friction $C_{f}$ increases on increasing thermal Grashof number $G_{r}$, solutal Grashof number $G_{m}$, Prandtl number $P_{r}$ and radiation parameter $N$ whereas it decreases on increasing the Darcy parameter $D a$, Magnetic parameter $M$, Schmidt number $S_{c}$, Soret number $S_{r}$, chemical reaction parameter $K_{r}$ and cold wall slip parameter $\gamma$ at both cold and heated walls. Skin friction coefficient $C_{f}$ decreases at the cold wall and increases at the heated wall on increasing the pressure gradient $\lambda$ and heated wall slip parameter $\sigma$. It is also noted that the skin friction coefficient attain their steady state for large time $\tau$.

The numerical values of heat transfer coefficient $N u$, obtained from the exact analytical solution, presented by equation (41), are presented in tabular form in table 4. It is clear that, the Nusselt number $\mathrm{Nu}$ decreases at the cold wall and increases at the heated wall on increasing the Prandtl number $P_{r}$ and radiation parameter $N$. It is clear that the heat transfer coefficient attains their steady state for large time $\tau$. Also the value of $N u$ is least for mercury and highest for water at $4{ }^{\circ} \mathrm{C}$.

The numerical values of mass transfer coefficient $S h$, obtained from the exact analytical solution, presented by equation (42), are presented in tabular form in tables 5 to 6 . It is clear that, Sherwood number $S h$ decreases at the cold wall and increases at the heated wall on increasing the Prandtl number $P_{r}$, radiation parameter $N$, Schmidt number $S_{c}$, Soret number $S_{r}$ and chemical reaction parameter $K_{r}$. It is clear that the mass transfer coefficient attains their steady state for large time $\tau$.

\section{Conclusions}

The influence of Schmidt number, Soret number, chemical reaction parameter, Prandtl number, thermal radiation parameter, pressure gradient, thermal Grashof number, solutal Grashof number, 
Magnetic parameter and Darcy parameter on unsteady hydromagnetic flow with heat and mass transfer of a viscous, incompressible, electrically conducting fluid past a parallel plate channel embedded in a fluid saturated porous medium is carried out. From the present investigation the following conclusions can be drawn:

$\bullet$

randtl number, radiation parameter, thermal Grashof number, Solutal Grashof number and pressure gradient are tends to accelerate fluid velocity whereas Schmidt number, Soret number, chemical reaction parameter, magnetic parameter and Darcy parameter have reverse effect on it throughout the boundary layer region.

elocity decreases in a region near to the plate and increases in a region away from the plate on increasing the heated wall slip parameter. Cold wall slip parameter tends to accelerate the fluid velocity in a region close to the plate whereas it has a reverse effect in a region away from the plate.

hermal radiation parameter and Prandtl number are tends to accelerate fluid temperature throughout the boundary layer region. hemical reaction parameter, Soret number, Schmidt number, thermal radiation parameter and Prandtl number are tend to retard the species concentration throughout the boundary layer region.

$\bullet$

hermal Grashof number, Solutal Grashof number, Prandtl number and thermal radiation parameter are tends to accelerate the skin friction coefficient whereas Darcy parameter, magnetic parameter, Schmidt number, Soret number, chemical reaction parameter and cold wall slip parameter have a reverse effect on the skin friction coefficient. Pressure gradient and heated wall slip parameter are tends to decelerate the skin friction coefficient at the cold wall whereas it is observed that reverse effect at the heated wall.

eat transfer coefficient decreases at the cold wall and increases at the heated wall on increasing the thermal radiation parameter and Prandtl number.

ass transfer coefficient decreases at the cold wall and increases at the heated wall on increasing the chemical reaction parameter, Soret number, Schmidt number, radiation parameter and Prandtl number. 
elocity, temperature, concentration, skin friction coefficient, Nusselt number and Sherwood number attain their steady state with the progress of time.

\section{ACKNOWLEDGEMENTS}

First author is thankful to V. R. Siddhartha Engineering College, Kanuru, Vijayawada, Andhra Pradesh, India for providing necessary research facilities to publish this paper. Authors are thankful for the suggestions and comments of the referees, which have led to improvement of the paper. 


\section{REFERENCES}

[1] Abdul Hakeem A. K. and Sathiyanathan K: An analytic solution of an oscillatory flow through a porous medium with radiation effect, Nonlinear Analysis: Hybrid Systems, vol. 3(2009), pp. 288-295.

[2] Adesanya S. O and Ayeni R.O: Existence and uniqueness result for couple stress bio fluid flow model via adomian decomposition method, Int. J. Nonlinear Science, vol. 12(2011), pp. 16- 24.

[3] Adesanya S. O and Makinde O. D: MHD oscillatory slip flow and heat transfer in a channel filled with porous media, U.P.B. Sci. Bull., Series A, vol. 76, no. 1(2014), pp.197-204.

[4] Derek C, Tretheway D. C and Meinhart C. D: Apparent fluid slip at hydrophobic microchannel walls, Phys. Fluids, vol.14 (2002), pp. L9-L12.

[5] Eegunjobi A. S and Makinde O. D: Combined effect of buoyancy force and navier slip on entropy generation in a vertical porous channel, entropy, vol. 14 (2012), pp 1028-1044.

[6] El-Shahed M: Pulsatile flow of blood through a stenosed porous medium under periodic body acceleration, Applied Mathematics and Computation, vol.138 (2003), pp.479-488.

[7] Eldabe N. T. M, Hassan A. A and Mohamed M. A. A: Effect of couple stresses on the MHD of a nonnewtonian unsteady flow between two parallel porous plates, Z. Naturforsch, vol. 58a (2003), pp. 204 210.

[8] Jha B. K and Ajibade A. O: Effect of viscous dissipation on natural convection flow between vertical parallel plates with time-periodic boundary conditions, Commun Nonlinear Sci Numer Simulat, vol.17 (2012), pp. 1576-1587.

[9] Jha B. K. and Ajibade A. O: Free convective flow between vertical porous plates with periodic heat input, Z. Angew. Math. Mech., (2010), pp.1-9

[10] Jha B. K. and Ajibade A. O: Free convective flow of heat generating/absorbing fluid between vertical porous plates with periodic heat input, Int. Commun. Heat and Mass Transf, vol. 36 (2009), pp. 624631.

[11] Khaled A. R. A and Vafai K: The effect of the slip condition on stokes and coutte flows due to an oscillating wall: exact solutions, Int. J. Nonlinear Mech, vol. 39(2004), pp. 795-809.

[12] Makinde O.D and Mhone P. Y: Heat transfer to MHD oscillatory flow in a channel filled with porous medium, Rom. Journ. Phys., vol. 50 (2005), pp. 931-938

[13] Makinde O. D and Osalusi E: MHD Steady flow in a channel with slip at the permeable boundaries, Rom. J. Phys, vol. 51(3-4) (2006), pp. 319-328.

[14] Marques Jr. W, Kremer G. M and Shapiro F. M: Coutte flow with slip and jump boundary conditions, Continumm Mech. Thermodynam, vol.12 (2000), pp.379-386.

[15] Manjula J, Padma P, Gnaneswara Reddy M and Venakateswarlu M: Influence of thermal radiation and chemical reaction on MHD flow, heat and mass transfer over a stretching surface, Procedia Engineering, vol.127 (2015), pp. 1315-1322.

[16] Massoudi M and Phuoc T. X: Pulsatile flow of blood using a modified second-grade fluid model, Compt and Maths with Applications, vol 56 (2008), pp. 199-211.

[17] Mehmood A and Ali A: The effect of slip condition on unsteady MHD Oscillatory flow of a viscous fluid in a planer channel, Rom. Journ. Phys, vol. 52 (2007), pp. 85-91.

[18] Mistra J. C and Pal B: A mathematical model for the study of the pulsatile flow of blood under an externally imposed body acceleration, Mathematical and Computer Modelling vol. 29 (1999), pp.89106.

[19] Navier C. L. M. H: Memoire Surles du Movement des, Mem Acad. Sci. Inst. France, vol. 1, No. 6(1823), pp. 414-416.

[20] Rathod V. P and Tanveer S: Pulsatile flow of couple stress fluid through a porous medium with periodic body acceleration and magnetic field, Bull. Malays. Math. Sci. Soc. vol. 32 (2009), pp. 245259. 
[21] Ruckenstein E and P. Rajora P: On the no-slip boundary conditions of hydrodynamics, J. Colloid Interface Sci, vol. 96 (1983), pp. 488-491.

[22] Sankar D. S and Lee U: Two-fluid Casson model for pulsatile blood flow through stenosed arteries: A theoretical model, Commun Nonlinear Sci Numer Simulat, vol.15 (2010), pp. 2086-2097.

[23] Soltani F and Yilmazer U: Slip velocity and slip layer thickness in flow of concentrated suspensions, $J$. Appl. Polym. Sci, vol. 70 (1998), pp. 515-522.

[24] Umuvathi J. C, Chamkha A. J, Mateen A, and Al-Mudhaf A: Unsteady oscillatory flow and heat transfer in a horizontal composite porous medium channel, Nonlinear analysis: Modelling and control, vol.14 (2009), pp. 397-415.

[25] Venkateswarlu M and Padma P: Unsteady MHD free convective heat and mass transfer in a boundary layer flow past a vertical permeable plate with thermal radiation and chemical reaction, Procedia Engineering, vol.127 (2015), pp. 791-799.

[26] Venkateswarlu M, Ramana Reddy G. V and Lakshmi D. V: Radiation effects on MHD boundary layer flow of liquid metal over a porous stretching surface in porous medium with heat generation, J. Korean Soc. Ind. Appl. Math., vol. 19, no. 1(2015), pp.83-102.

[27] Venkateswarlu M, Ramana Reddy G. V and Lakshmi D. V: Thermal diffusion and radiation effects on unsteady MHD free convection heat and mass transfer flow past a linearly accelerated vertical porous plate with variable temperature and mass diffusion, J. Korean Soc. Ind. Appl. Math., vol. 18, no. 3(2014), pp. 257-268.

[28] Venkateswarlu M, Ramana Reddy G. V and Lakshmi D. V: Diffusion-thermo effects on MHD flow past an infinite vertical porous plate in the presence of radiation and chemical reaction, International Journal of Mathematical Archive, vol. 4, no. 9(2013), pp. 39-51.

[29] Watanebe K, Yanuar and Mizunuma H: Slip of Newtonian fluids at solid boundary, JSME Int. J. Ser. B, vol. 41(1998), pp. 525-529.

[30] Yu S and Ameel T. A: Slip-flow heat transfer in rectangular microchannels, Int. J. Heat Mass Transfer, vol. 44 (2002) pp. 4225-4234.

[31] Zuezo J and Beg O. A: Network numerical simulation applied to pulsatile non-Newtonian flow through a channel with couple stress and wall mass flux effects, Int. J. of Applied Math and Mech., vol.5 (2009), pp.1-16. 


\section{APPENDIX}

$$
\begin{aligned}
& m_{1}=\sqrt{N P_{r}}, m_{2}=\sqrt{P_{r}(N-i \omega)}, m_{3}=\sqrt{S_{c} K_{r}}, A_{1}=S_{c} S_{r}, A_{2}=\frac{m_{1}^{2} A_{1}}{m_{1}^{2}+m_{3}^{2}}, A_{3}=1+A_{2}, \\
& m_{4}=\sqrt{S_{c}\left(K_{r}+i \omega\right)}, A_{4}=\frac{m_{2}^{2} A_{1}}{m_{2}^{2}+m_{4}^{2}}, A_{5}=1+A_{4}, m_{5}=\sqrt{M+\frac{1}{D_{a}}}, A_{6}=\frac{G_{r}-G_{m} A_{2}}{m_{1}^{2}+m_{5}^{2}}, \\
& A_{7}=\frac{G_{m} A_{3}}{m_{3}^{2}-m_{5}^{2}}, A_{8}=\frac{\lambda_{0}}{m_{5}^{2}}, A_{9}=\frac{\gamma m_{1} A_{6}}{\sin \left(m_{1}\right)}, A_{10}=\frac{\gamma m_{3} A_{7}}{\sinh \left(m_{3}\right)}, A_{11}=A_{9}-\left(A_{8}+A_{10}\right), \\
& A_{12}=1-\sigma m_{3} \operatorname{coth}\left(m_{3}\right), A_{13}=1-\sigma m_{1} \cot \left(m_{1}\right), A_{14}=A_{7} A_{12}-\left(A_{8}+A_{6} A_{13}\right), \\
& A_{15}=1+\gamma m_{5}, A_{16}=1-\gamma m_{5}, A_{17}=1+\sigma m_{5}, A_{18}=1-\sigma m_{5}, A_{19}=A_{11} A_{17} \exp \left(-m_{5}\right)-A_{14} A_{15}, \\
& A_{20}=A_{16} A_{17} \exp \left(-m_{5}\right)-A_{15} A_{18} \exp \left(m_{5}\right), A_{21}=\frac{A_{19}}{A_{20}}, A_{22}=\frac{A_{11}-A_{16} A_{21}}{A_{15}}, \\
& m_{6}=\sqrt{M+\frac{1}{D_{a}}+i \omega, A_{23}=} \frac{G_{r}-G_{m} A_{4}}{m_{2}^{2}+m_{6}^{2}}, A_{24}=\frac{G_{m} A_{5}}{m_{4}^{2}-m_{6}^{2}}, A_{25}=\frac{\lambda_{1}}{m_{6}^{2}}, A_{26}=1+\gamma m_{6}, \\
& A_{27}=1-\gamma m_{6}, A_{28}=1+\sigma m_{6}, A_{29}=1-\sigma m_{6}, A_{30}=\frac{\gamma m_{2} A_{23}}{\sin \left(m_{2}\right)}, A_{31}=\frac{\gamma m_{4} A_{24}}{\sinh \left(m_{4}\right)}, \\
& A_{32}=A_{30}-\left(A_{25}+A_{31}\right), A_{33}=1-\sigma m_{4} \operatorname{coth}\left(m_{4}\right), A_{34}=1-\sigma m_{2} \cot \left(m_{2}\right), \\
& A_{35}=A_{24} A_{33}-\left(A_{25}+A_{23} A_{34}\right), A_{36}=A_{27} A_{28} \exp \left(-m_{6}\right)-A_{26} A_{29} \exp \left(m_{6}\right), \\
& A_{37}=A_{28} A_{32} \exp \left(-m_{6}\right)-A_{26} A_{35}, A_{38}=\frac{A_{37}}{A_{36}}, A_{39}=\frac{A_{32}-A_{27} A_{38}}{A_{26}}
\end{aligned}
$$




\section{NOMENCLATURE}

$\begin{array}{ll}B_{0} & \text { uniform magnetic field } \\ C & \text { species concentration } \\ C_{f} & \text { skin-friction coefficient } \\ C_{1} & \text { species concentration at the heated wall } \\ C_{0} & \text { species concentration at the cold wall } \\ C_{p} & \text { specific heat at constant pressure } \\ D_{m} & \text { chemical molecular diffusivity } \\ G_{m} & \text { Solutal Grashof number } \\ G_{r} & \text { thermal Grashof number } \\ g & \text { acceleration due to gravity } \\ j_{w} & \text { mass flux } \\ D a & \text { Darcy parameter } \\ K & \text { permeability of porous medium } \\ K_{r}^{*} & \text { dimensional chemical reaction } \\ K_{r} & \text { chemical reaction parameter } \\ K_{T} & \text { thermal conductivity of the fluid } \\ M & \text { magnetic parameter } \\ N & \text { radiation parameter } \\ N u & \text { Nusselt number } \\ n & \text { frequency of oscillation } \\ P_{r} & \text { Prandtl number } \\ q_{w} & \text { heat flux } \\ S_{c} & \text { Schmidt number } \\ S h & \text { Sherwood number } \\ S_{r} & \text { Soret number } \\ T & \text { fluid temperature } \\ & \end{array}$

\author{
$T_{m} \quad$ mean temperature of the fluid \\ $T_{1} \quad$ fluid temperature at the heated wall \\ $T_{0} \quad$ fluid temperature at the cold wall \\ $t \quad$ dimensional time \\ $U \quad$ A scaled velocity \\ $u \quad$ fluid velocity in $x$ direction \\ $v \quad$ fluid velocity in $y$ direction
}

\section{Greek Symbols}

$\alpha \quad$ dimensional thermal radiation parameter

$\beta_{\mathrm{c}} \quad$ coefficient expansion for species concentration

$\beta_{\mathrm{T}} \quad$ coefficient of thermal expansion

$\mathrm{v} \quad$ kinematic coefficient of viscosity

$\omega \quad$ A scaled frequency

$\phi \quad$ A scaled concentration

$\phi_{1} \quad$ dimensional cold wall slip parameter

$\phi_{2} \quad$ dimensional heated wall slip parameter

$\rho \quad$ fluid density

$\sigma_{\mathrm{e}} \quad$ electrical conductivity

$\tau$ non dimensional time

$\tau_{\mathrm{w}} \quad$ shear stress

$\eta \quad$ A scaled coordinate

$\theta \quad$ A scaled temperature

$\gamma \quad$ non-dimensional cold wall slip parameter

$\sigma \quad$ non-dimensional heated wall slip parameter 